\title{
EXPERIENCIAS EDUCATIVAS BASADAS EN EL SISTEMA MARPABIOBOT
}

\author{
Gustavo A. Casañ \\ IRS Lab, Universitat Jaume I, 12071, ncasan@uji.es \\ José Vicente Martí \\ IRS Lab, Universitat Jaume I, 12071, vmarti@uji.es \\ Raúl Marín \\ IRS Lab, Universitat Jaume I, 12071, rmarin@uji.es \\ David Fornás \\ IRS Lab, Universitat Jaume I, 12071, dfornas@uji.es \\ Pedro J. Sanz \\ IRS Lab, Universitat Jaume I, 12071, sanzp@uji.es
}

\begin{abstract}
Resumen
En este articulo se presenta un robot modular y de bajo coste, MARPABIOBOT (Modular Arduino ROS Printable Android Robot), que ya ha sido puesto a prueba con éxito como herramienta educativa en varios niveles educativos (colegio, instituto y universidad). Al ser hardware y software libre, basado en componentes de bajo coste y fácil accesibilidad, las posibilidades de aprendizaje que ofrece destacan sobre las de otros sistemas robóticos que están más orientados al aprendizaje de uno de los aspectos de la robótica (generalmente la programación) o limitan mucho la profundidad a la que se puede llegar al emplearlos.
\end{abstract}

Palabras clave: Robótica educativa, robots móviles, robótica libre.

\section{INTRODUCCIÓN}

Utilizar robots en educación tiene una larga historia [16] [10] [2], dado que han demostrado ser una buena herramienta para aprender mecánica, diseño, electrónica y programación [32] [28] [30] [33] [35] y se enmarca dentro del popular movimiento STEM [4]. Sin embargo, también desde el principio, la mayoría de los centros, incluso universitarios, no podían permitirse el precio de un robot (siendo impensable tener robots para todos los alumnos de un grupo). Afortunadamente en los últimos años se ha producido una proliferación de robots de coste relativamente reducido (por ejemplo, los Mindstorm de LEGO o el popular robot e-puck [25]) que permiten hacer accesible a un público más amplio el campo de la robótica. Al mismo tiempo, los simuladores (como USARSim (Unified System for Automation and Robot Simulation) [5], Webots [23] o SimSpark [34]) y laboratorios remotos (como el telerrobot de la Universidad de Australia Occidental (UWA) [11], el Laboratorio Remoto PR2 [26] o RPN [7]) se han hecho también más accesibles y demostrado su utilidad [8] [9], o el UJI Online Robot [20].

En este artículo se presenta un robot modular y de bajo coste, MARPABIOBOT, que ya ha sido puesto a prueba como herramienta educativa en diversos ámbitos. Inicialmente se mostrará un pequeño estado del arte de la robótica educativa de bajo coste para posteriormente hablar de la motivación para el desarrollo del robot, sus características y el desarrollo de las experiencias de aprendizaje. Para finalizar se expondrán unas conclusiones y posibilidades de trabajo futuro del proyecto.

\section{ESTADO DEL ARTE}

Existe una cantidad tan grande de robots distintos que se emplean con propósitos educativos que resulta imposible nombrarlos todos. La mayoría se centran en el robot como un apoyo físico para el aprendizaje de programación y en muchos casos utilizan lenguajes (entornos) de programación visuales como Scratch [30]. A continuación, se muestra una selección de los que se consideran más representativos:

Lego [18] [22] produce varios robots modulares que en teoría se pueden combinar para formar distintos modelos. Algunos de ellos están más 
orientados al entretenimiento, pero hay una importante línea de material educativo (no sólo robots). Al ser compatible con las piezas estándar de Lego su forma puede ser bastante variada y hay bastante material educativo asociado a cada modelo. Los problemas que presentan están relacionados con la funcionalidad y el precio, superior al de otros sistemas de prestaciones similares.

El robot e-puck [25] [13] es un sencillo robot móvil con una batería de vida muy larga. Es tan popular que existe una nueva versión, e-puck2 desde 2018. Hay varias extensiones disponibles del robot, pero no dispone de manipuladores, lo cual lo limita.

El robot BIOLOID [3] es un paquete robótico producido por el fabricante ROBOTIS. La plataforma consiste de componentes y pequeños servomecanismos 1(e.g. AX-12A Dynamixel), que pueden usarse para construir robots de varias configuraciones, con ruedas, piernas o humanoides. El robot se programa con RoboPlus, una herramienta que incluye la edición de movimientos y scripts, así como la configuración de los motores. La rama Bioloid es utilizada habitualmente en las competiciones de robots humanoides (e.g. CEABOT, [6]), pues permite conectar en cadena un gran número de servo motores.

Makeblock [19] toma como base la programación de dispositivos Arduino [1] adaptados para estudiantes de diferentes edades. Los conectores utilizados (RJ-25) son un buen ejemplo de esta mejora, así como la conexión bluetooth y su programación a través de un dispositivo Android. Es destacable también el gran número de piezas mecánicas en aluminio, lo cual da un acabado profesional a los robots.

Además de Makeblock existen multitud de robots basados en Arduino, pero su alcance es limitado dado que, en muchos casos, se trata casi de un "hazlo tú mismo" completo, y el proceso de aprendizaje se produce en la construcción y montaje del robot, no en su uso posterior. Y por supuesto existen robots educativos más específicos, como drones o robots submarinos (como el MIT Sea Pearch [24]).

Sin embargo, la mayoría de estos robots son muy distintos de los robots empleados en la industria o la investigación, que son sistemas únicos o emplean el sistema operativo ROS [27], mientras que mecánicamente se emplean un gran número de sistemas neumáticos, algo que apenas se ve en los sistemas educativos.

\section{MOTIVACIÓN}

La robótica es una disciplina que aglutina muchas ciencias y tecnologías y a la que uno puede aproximarse desde muchas perspectivas distintas. Así, se puede practicar robótica centrándonos en la programación de robots ya existentes, prácticamente cajas negras para el usuario, pero también se puede practicar la robótica desde el diseño mecánico de los componentes, la integración de componentes ya existentes o la creación de nuevos sensores. La mayor parte de robots educativos (especialmente los orientados hacia los niveles más básicos) se centran en los aspectos de programación de un robot ya existente, ofreciendo algunos la posibilidad de incorporar módulos ya existentes de una forma muy controlada (tal vez el ejemplo más famoso de esto sean los robots de Lego).

Aunque estos son una gran herramienta para la enseñanza de la programación se quedan cortos como herramientas para enseñar una robótica completa, dado que el diseño de partes y su integración son bastante dificultosos. Además, en muchos casos, utilizan software propio (sistema operativo y lenguaje de programación), por lo que lo aprendido no tiene una aplicación inmediata fuera del robot concreto empleado.

Existe una necesidad de crear sistemas que permitan practicar el diseño 3D y la programación Linux/ROS, para la navegación y manipulación robótica, para su aplicación tanto en institutos como en asignaturas de Universidad. $\mathrm{Si}$ a esto le añadimos la posibilidad de practicar la programación Android y la programación Arduino tenemos una herramienta muy adecuada para el aprendizaje.

El uso de robots hace que el aprendizaje de programación sea más atractivo, mientras que, por otro lado, la posibilidad de programar el robot proporciona un conocimiento más profundo acerca de su funcionamiento. Los robots ya se han utilizado para facilitar el proceso de aprendizaje de los cursos introductorios de programación [10] [11]. Los robots ofrecen a los estudiantes de programación un modelo físico para demostrar visualmente conceptos o ideas enseñadas tradicionalmente usando abstracciones.

\section{MARPABIOBOT}

El MARPABIOBOT es un robot móvil que emplea una placa Arduino como procesador, que además puede controlarse a través de Bluetooth con un ordenador con ROS instalado (en la Figura 1 puede 
verse la arquitectura del software empleada), incluyendo un sistema de motores y ruedas para desplazarse, y una garra simple para la manipulación de objetos.

El robot incorpora los dos servomotores de tracción (izquierdo -pin 10- y derecho -pin 9-), el servomotor que sirve para orientar el medidor de distancias (pin 11), el propio medidor de distancias (entrada 0 para medición $\mathrm{A} / \mathrm{D}$, pin 3 salida para producir medición y pin 2 entrada para recibir el eco de la medición) $y$, algunos de ellos, la pinza (pin 5). Además el robot está preparado para incluir un dispositivo Android (aunque no es imprescindible), sobre todo para aplicaciones de visión a través de ROS. En la Figura 2 puede verse el robot conectado a un teléfono móvil y en la dirección [15] un vídeo del robot en movimiento. Dado el uso habitual de los móviles hoy en día reutilizar un modelo "viejo" permite mantener el bajo coste del robot.

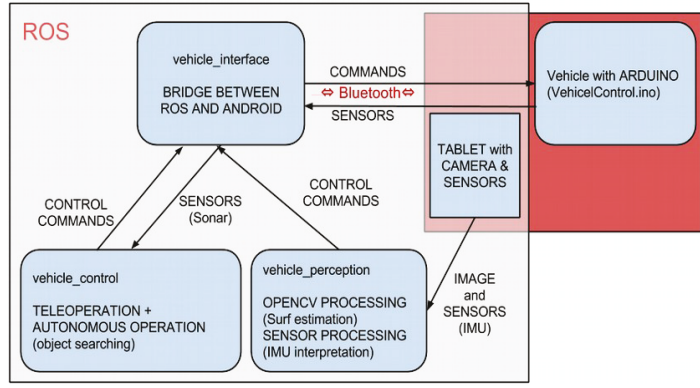

Figura 1: Arquitectura del software.

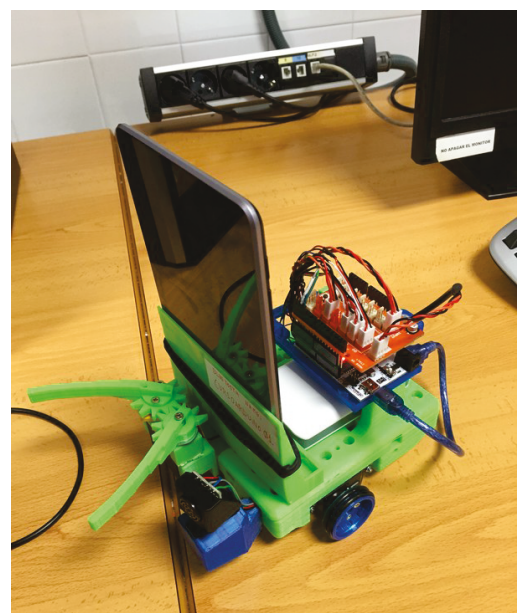

Figura 2: Robot MARPABIOBOT.

Entre sus características más importantes vale la pena destacar la posibilidad de integrarlo con un teléfono móvil con Android y aprovechar todas las capacidades de éste (micrófono, cámara, GPS, altavoz...) y, por otra parte, el hecho de que el diseño 3D de las piezas incluya un acople estándar a partir del cual cualquiera puede diseñar una pieza que se integrará sin problemas en el robot. En la Figura 3 pueden verse los anclajes, macho y hembra, usados para unir cualquier sensor $\mathrm{o}$ dispositivo al cuerpo del robot.

Figura 3: Anclajes empleados en el robot.

\section{EXPERIENCIAS REALIZADAS}

Las primeras pruebas del robot se iniciaron en la Escuela de Verano de la Universidad Jaume I, abierta a niños de entre 4 y 16 años (divididos según edades) en las cuales, junto a otras actividades educativas y recreativas, se les ofrecía la posibilidad de aprender robótica. Mientras que para los más pequeños las actividades eran básicamente de observación, los más mayores ya se iniciaban en la programación y el diseño de pruebas para los robots (explorar un espacio desconocido evitando obstáculos, localizar una vela encendida y acercarse a ella). La respuesta, cómo no, fue positiva.

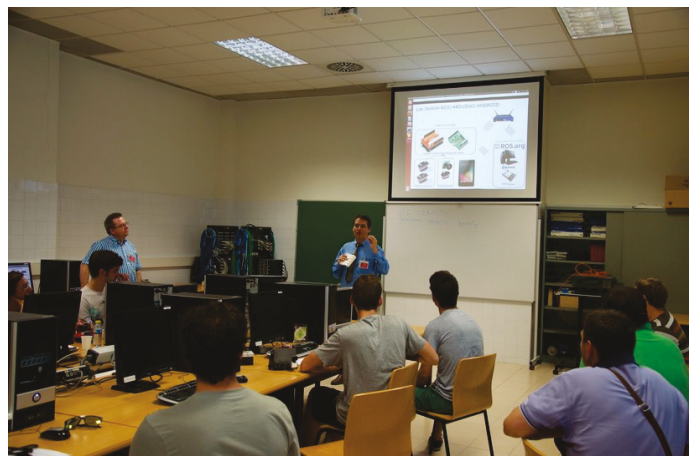

Figura 4: Summer School on Mobile Manipulators 2015.

Una experiencia significativa fue la utilización del robot en una sesión práctica de la escuela de verano 2015 Summer School on Mobile Manipulators [31] con el título Control \& Navigation of a Real Robotic Platform impartida por los profesores Raúl Marín y José Vicente Martí. El perfil de los estudiantes era internacional y especializado, siendo la mayoría de ellos estudiantes de doctorado. $\mathrm{Su}$ tarea era programar un controlador para que el 
robot se desplazase con libertad por un entorno desconocido (un laberinto) hasta localizar un objeto de color rojo, se aproximasen a él y lo recogiesen, volviendo al punto inicial. En la Figura 4 puede verse una imagen de este encuentro.

En el Grado de Informática de la Universidad Jaume I el MARPABIOBOT se ha empleado para promocionar la intensificación de Ingeniería de Computadores mediante la organización de cursos de robótica elemental e IoT (Internet of Things, Internet de las cosas) para estudiantes de los dos primeros cursos del Grado (Figura 5). También es de destacar su uso en la asignatura "Redes y Dispositivos Móviles", optativa de cuarto curso del Grado de Informática, como ejemplo de la programación de un dispositivo Arduino remoto [29], acoplando un teléfono móvil Arduino al robot $\mathrm{y}$ empleando sus sensores y capacidades para resolver tareas sencillas, como desplazarse a unas coordenadas GPS evitando obstáculos o moverse hacia el sonido más fuerte.

Asimismo, se emplea habitualmente en el Máster en Sistemas Inteligentes [21] en la realización de trabajos final de máster por parte de los estudiantes, empleando las capacidades de sensorización, comunicación y control remoto de los robots. También se emplean para proyectos en las asignaturas "Percepción y manipulación" y "Robótica Cooperativa", en la que los estudiantes deben programar varios robots para que se comuniquen y localicen más rápidamente objetos en entornos desconocidos.

Especial mención debe hacerse de su uso en el máster interuniversitario Erasmus Mundus Advanced Robotics, EMARO [12] en la que estos robots se emplean en proyectos individuales y de grupo en la asignatura "Telerobotics".

Figura 5: Convocatoria de taller de Robótica para IoT para estudiantes del grado de Informática.

El perfil de los alumnos puede dividirse en tres grandes grupos:
- Estudiantes de colegio e instituto que realizan la actividad como parte de actividades en parte recreativas.

- Estudiantes universitarios del grado en informática, en su mayor parte españoles y cuyo trabajo va a ser evaluado en cuanto al diseño de aplicaciones Android y Arduino.

- Estudiantes de máster en sistemas inteligentes y en robótica, con un marcado carácter internacional y clases en inglés en su mayor parte. También se produce una evaluación del trabajo realizado.

En todos los casos la respuesta de los alumnos ha sido muy positiva, permitiéndoles interactuar con el dispositivo a diversos niveles y estimulando su interés por la robótica.

Por último mencionar que el tipo de actividades que tienen que realizar es el clásico con este tipo de robots móviles con sensores y manipuladores: programar al robot para explorar un espacio, recorrer un laberinto reconociendo objetos, localizar un objeto y trasladarlo a otro predeterminado... Excepto por pequeñas modificaciones en las pinzas y el montaje del robot desde cero todavía no se han explorado en profundidad las posibilidades del diseño 3D. Por otra parte si que se han empleado herramientas básicas de ludoficación (gamificación) [17] al organizar pequeñas competiciones internas entre los estudiantes parra ver quién lograba el robot que acabase antes una tarea (recorrer un laberinto o localizar un objeto, por ejemplo).

\section{CONCLUSIONES}

El robot presentado ha demostrado su adecuación a las tareas de enseñanza de la robótica en diversos contextos educativos. Sus características le convierten en una opción válida para la enseñanza de los aspectos menos trabajados de la robótica, como el diseño y la mecanización, gracias a la combinación de reducido precio de los componentes, software libre y el uso de impresión 3D. Los diseños y software son públicos y de uso libre para enseñanza e investigación (y accesibles en el repositorio [14]). Remarcar su papel como herramienta de enseñanza de IoT, que se sale de lo habitual en un sistema robótico educativo.

Como extensiones futuras hay varios aspectos en los que se planea orientar el desarrollo del proyecto, como pueden ser la integración de herramientas de programación gráfica como Scratch o Blockly en el entorno, la conexión con 
otros tipos de sensores y el diseño de piezas complementarias imprimibles en 3D.

\section{Agradecimientos}

Este trabajo ha sido parcialmente financiado por el Ministerio de Economía, Industria y Competitividad de España con la subvención "DPI2017-86372-C3-1-R" (Proyecto COMOMUIS), por el Gobierno de la Comunidad Valenciana bajo las subvenciones "GVAPROMETEO/2016/066" e "IDIFEDER/2018/013", y por la UJI bajo la subvención "UJI B2018-34" (Proyecto NEPTUNO).

\section{English summary}

\section{EDUCATIVE EXPERIENCES BASED ON THE MARPABIOBOT SYSTEM}

\begin{abstract}
In this paper MARPABIOBOT, a modular low cost robot which has already been tested as a teaching tool at several educational levels (school, high school and university) is presented. As it is free hardware and software, based in low cost and easy to find and use components, the possibilities it offers stand out among other robotic systems that are usually oriented towards only one aspect of robotics (generally programming) or they are limited in the depth they allow the students to arrive.
\end{abstract}

Keywords: Robotics in education, mobile robots, open robotics.

\section{Referencias}

[1] Arduino, https://www.arduino.cc/. Consultado 15 de mayo de 2019.

[2] Barreto, F. \& Benitti, V. (2012). Exploring the educational potential of robotics in schools: A systematic review. Computers \& Education, volume 58, issue 3, pages 978988.

[3] BIOLOID. http://www.robotis.us/bioloid-1/. Consultado 1 de mayo 2019.

[4] Breiner, J. M., Harkness, S. S., Johnson, C. C. y Koehler, C. M. (2012). What Is STEM?
A Discussion About Conceptions of STEM in Education and Partnerships, School Science and Mathematics, Wiley Online Library, issn 1949-8594, doi: 10.1111/j.1949-8594.2011.00109.x, vol. 112 (1), pp. 3-11.

[5] Carpin, S., Lewis, M., Wang, J., Balakirsky, S., Scrapper, C., (2007) Usarsim: a robot simulator for research and education. In: Robotics and Automation, 2007 IEEE International Conference on, pp. 1400-1405.

[6] CEABOT (2019). http://www.ceabot.es/ Consultado el 4 de junio de 2019.

[7] Cervera, E., Martinet, P., Marin, R., Moughlbay, A. Abou, del Pobil, A. P., Alemany, J., Esteller-Curto, R., and Casañ, G. A.: The Robot Programming Network. Journal of Inteligent and Robotic Sytems, 2015.

[8] D’Angelo, C., Rutstein, D., Harris, C., Bernard, R., Borokhovski, E., Haertel, G. (2014). Simulations for STEM Learning: Systematic Review and Meta-Analysis. Menlo Park, CA: SRI International.

[9] Dagdilelis, V., Sartatzemi, M., Kagani, K.: Teaching (with) robots in secondary schools: some new and not-so-new pedagogical problems. In: Advanced Learning Technologies, 2005. ICALT 2005. Fifth IEEE International Conference on, pp. 757761,2005

[10] De Jong, T., Linn, M.C. \& Zacharia, Z. C. (2013). Physical and Virtual Laboratories in Science and Engineering Education. Science, Volume 340, Issue 6130.

[11] Djalic, V., Maric, P., Kosic, D., Samuelsen, D., Thyberg, B. \& Graven, O. (2012). Remote laboratory for robotics and automation as a tool for remote access to learning content. Proc. 15th International Conference on Interactive Collaborative Learning, ICL 2012. Villach, Austria. DOI: 10.1109/ICL.2012.6402174 .

[12] EMARO. https://master-emaro.ec-nantes.fr/ Consultado el 1 de mayo de 2019.

[13] e-puck. http://www.e-puck.org/ Consultado el 1 de mayo de 2019.

[14] IRS Lab (2019). https://github.com/ujiirslab-repo/MARPABIOBOT. Consultado el 
3 de junio de 2019.

[15] IRS Lab (2019b). https://youtu.be/G28iqAvUSiQ Consultado el 9 de julio de 2019.

[16] Johnson, J. (2003). Children, robotics, and education. Artificial Life and Robotics, volume 7, issue 1, pages 16-21.

[17] Kapp, K. M. (2012). The gamification of learning and instruction: game-based methods and strategies for training and education. John Wiley \& Sons.

[18] Lawhead, P.B., Duncan, M.E., Bland, C.G., Goldweber, M., Schep, M., Barnes, D.J., Hollingsworth, R.G., (2003) A road map for teaching introductory programming using lego mindstorm robots. ACM SIGCSE Bulletin 35(2), 191-201.

[19] Makebot, https://www.makeblock.com/ Consultado 2 de mayo de 2019.

[20] Marín, R., Sanz, P. J., Pobil, A. (2002). The UJI Online Robot: An Education and Training Experience: Special Issue on Internet and Online Robots in Autonomous Robots Journal 15 (3), 2003. DOI: 10. 1023/ A:1026220621431.

[21] Máster en Sistemas Inteligentes. https://www.uji.es/estudis/oferta/base/master s/actual/centre/estce/sistemes-inteligents2013/ Consultado 1 de mayo de 2019.

[22] Mindstorms. https://www.lego.com/es-es/mindstorms Consultado 1 de mayo de 2019.

[23] Michel, O. (2004). Webots: Professional Mobile Robot Simulation. International Journal of Advanced Robotic Systems, volume 1, issue 1, pages 39-42.

[24] MIT Sea Pearch, https://seaperch.mit.edu/ Consultado 1 de mayo de 2019.

[25] Mondada, F., Bonani, M., Raemy, X., Pugh, J., Cianci, C., Klaptocz, A., Magnenat, S., Zufferey, J-C., Floreano, D. \& Martinoli, A. (2009). The e-puck, a Robot Designed for Education in Engineering. Proc. 9th Conference on Autonomous Robot Systems and Competitions, vol.1, issue 1, pp. 59-65.

[26] Pitzer, B., Osentoski, S., Jay, G., Crick, C., Jenkins, O.C., (2012) PR2 Remote Lab: An environment for remote development and experimentation. In: Robotics and Automation (ICRA), IEEE International Conference on, pp. 3200-3205.

[27] Quigley, M., Conley, K., Gerkey, B., Faust, J., Foote, T., Leibs, J., Wheeler, R., Ng, A.Y., (2009) Ros: an open-source robot operating system. In: ICRA workshop on open source software, vol. 3, p. 5. Kobe.

[28] Ratcliff, C. C. \& Anderson, S. E. (2011). Reviving the turtle: Exploring the use of logo with students with mild disabilities. Computers in the Schools, 28(3), pp. 241255. DOI: 10.1080/07380569.2011.594987.

[29] Redes y Dispositivos Móviles. http:/ujiapps.uji.es/sia/rest/publicacion/2018/ estudio/225/asignatura/EI1057 Consultado 2 de mayo de 2019.

[30] Resnick, M., Maloney, J., MonroyHernández, A., Rusk, N., Eastmond, E., Brennan, K., Millner, A., Rosenbaum, E., Silver, J., Silverman, B. \& Kafai, Y. (2011). Scratch: Programming for all. Communications of the ACM, vol. 52 (11),

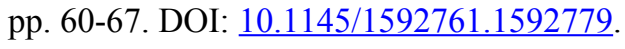

[31] Summer School on Mobile Manipulators 2015.

https://sites.google.com/a/uji.es/summerscho ol2015/home Consultado 3 de mayo de 2019.

[32] Schilling, K., Roth, H. \& Rösch, O. J. (2002). Mobile mini-robots for engineering education. Global Journal of Engineering Education, 6 (1), 79-84.

[33] Tillmann, N., Moskal, M., de Halleux, J., Fahndrich, M., Bishop, J., Samuel A. \& Xie, T. (2012). The future of teaching programming is on mobile devices. Proc. 17th ACM annual conference on Innovation and Technology in Computer Science Education, ITiCSE'12, 156-161. Haifa, Israel.

[34] Xu, Y., Vatankhah, H.: Simspark: an open source robot simulator developed by the robocup community. In: Robot Soccer World Cup, pp. 632-639. Springer (2013)

[35] Wang, E. (2001). Teaching freshmen design, creativity and programming with LEGOs and LabVIEW. Proc. 31st Annual Frontiers in Education Conference, FiE 2001, F3G-11. Reno, USA. 


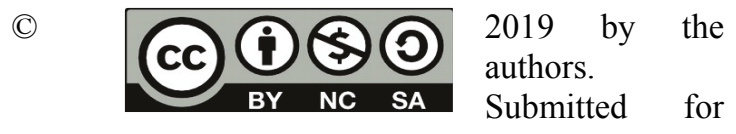
possible open access publication under the terms and conditions of the Creative Commons Attribution CC BY-NC-SA 4.0 license (https:// creativecommons.org/licenses/by-nc-sa/4.0/ 